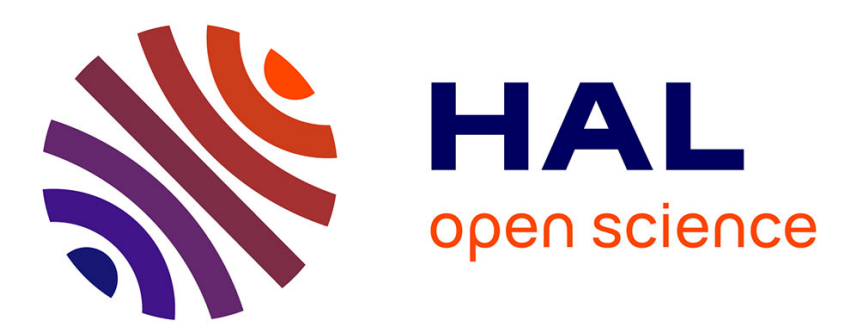

\title{
Les pays d'Europe Centrale et Orientale sont-ils fortement exposés aux chocs sectoriels et géographiques vis-à-vis de la zone euro? \\ Jérôme Trotignon
}

\section{- To cite this version: \\ Jérôme Trotignon. Les pays d'Europe Centrale et Orientale sont-ils fortement exposés aux chocs sectoriels et géographiques vis-à-vis de la zone euro ?. Revue d'Etudes Comparatives Est-Ouest, 2005, 36 (2), pp.109-141. halshs-00161687}

\section{HAL Id: halshs-00161687 \\ https://shs.hal.science/halshs-00161687}

Submitted on 27 Mar 2009

HAL is a multi-disciplinary open access archive for the deposit and dissemination of scientific research documents, whether they are published or not. The documents may come from teaching and research institutions in France or abroad, or from public or private research centers.
L'archive ouverte pluridisciplinaire HAL, est destinée au dépôt et à la diffusion de documents scientifiques de niveau recherche, publiés ou non, émanant des établissements d'enseignement et de recherche français ou étrangers, des laboratoires publics ou privés. 


\title{
Les Pays d'Europe centrale et orientale sont-ils fortement exposés aux chocs sectoriels et géographiques vis-à-vis de la zone euro ?
}

\author{
Jérôme Trotignon*
}

\section{Revue d'études comparatives Est-Ouest, vol. 36, $n^{\circ}$ 2, 2005}

\section{Résumé}

La future adhésion des PECO à la zone euro met en avant le risque de choc asymétrique externe, dû à une trop forte dépendance sur un secteur ou un pays client. Nous construisons deux indicateurs - sectoriel et géographique - d'exposition aux chocs, à partir de la symétrie des structures d'exportation d'un futur membre avec l'UEM et de l'ouverture commerciale du futur membre. Cela nous permet d'établir un classement des PECO en fonction de la réalisation du critère de Kenen reformulé, puis transposé à la diversité géographique des exportations. Les résultats, comparés à ceux des deux pays de l'UEM les plus exposés aux chocs sectoriel et géographique (la Finlande et l'Irlande), témoignent d'un risque généralement prononcé. Un inventaire des couples pays/branche et pays/destination susceptibles d'être à l'origine de chocs à fort impact macroéconomique montre que la Bulgarie et la Slovaquie, et plus encore l'Estonie et la Lettonie, concentrent les risques majeurs. Le processus de convergence réelle de la Bulgarie et de la Lettonie avec l'eurozone ne semblant pas être amorcé, ces deux économies pourraient envisager de reporter leur participation à l'UEM ou de créer préalablement un fonds de stabilisation conjoncturel. Cette recommandation est contraire à la thèse de l'endogénéité des ZMO, mise en avant lorsque le commerce intrabranche s'intensifie. Mais la hausse de la part du commerce intrabranche entre un PECO et l'UEM ne correspond pas nécessairement à une moindre exposition aux chocs étudiés.

Mots-clés : choc asymétrique externe, critère de Kenen, exposition aux chocs, pays d'Europe centrale et orientale, symétrie des structures d'exportation, zone monétaire optimale.

\section{Are the Central and Eastern European countries greatly exposed to sectoral and geographic shocks vis-à-vis the eurozone?}

The future membership of the ten CEECs in the eurozone involves the risk of external asymmetric shocks, due to too strong a dependence on one sector or one customer country. The purpose of this paper is to build two indicators - sector-based and geographic - of exposure to shocks, taking into account the symmetry of the export structures of a future member with the EMU and the trade openness of the future member. It enables to draw up a classification of the CEECs according to the fulfillment of the Kenen criterion, revised and transposed to the geographic variety of exports. The results, compared with those of the two countries of the EMU which are most sensitive to sector-based and geographic shocks (Finland and Ireland), testify to a generally pronounced exposure to shocks. An inventory of the pairs country/branch and country/destination likely to be at the origin of shocks with strong macroeconomic impact shows that Bulgaria and Slovakia, and even more Estonia and Latvia, are exposed to major risks. In so far Bulgaria and Latvia's real convergence process with the eurozone doesn't seem to have really started, these two economies might consider postponing their EMU membership, or creating a cyclical stabilization fund joining it. This recommendation contradicts the endogenous OCA theory, which is put forward when intra-industry trade intensifies. But the increase in the share of intra-industry trade between a CEEC and the EMU does not necessarily entail less exposure to the shocks studied.

Keywords : Central and Eastern European countries, exposure to shocks, external asymmetric shock, the Kenen criterion, optimum currency area, symmetry of export structures.

JEL : F15, F33, F36

*Maître de Conférences, GATE - Université Lyon 2, trotignon@gate.cnrs.fr 


\section{Les Pays d'Europe centrale et orientale sont-ils fortement exposés aux chocs sectoriels et géographiques vis-à-vis de la zone euro ?}

L'adhésion de 10 pays d'Europe centrale et orientale (PECO) à l'Union européenne (UE) ouvre la voie de leur participation à l'UEM. L'un des critères d'adhésion à l'UE est l'acceptation de l'acquis communautaire, qui les conduira à appliquer les dispositions du Traité de Maastricht concernant la transition vers l’UEM. L'entrée de ces pays dans la zone euro est principalement conditionnée par la réalisation de critères de convergence nominaux. Mais il n’existe aucun pré requis sur les critères réels traditionnels définissant l'optimalité d'une zone monétaire, qu'il s'agisse de critères permettant d'éviter des chocs asymétriques (comme la diversification sectorielle chez P. Kenen), ou de résorber des chocs asymétriques (comme la mobilité du travail chez R. Mundell). Les approches récentes de la théorie des zones monétaires optimales (ZMO), initiées par T. Bayoumi et B. Eichengreen (1993), qui évaluent la symétrie des chocs et la corrélation des cycles économiques entre pays membres d’une même union monétaire, ne sont pas non plus prises en compte.

Cette lacune pourrait être lourde de conséquences : en cas de choc asymétrique à fort impact macroéconomique, les PECO, une fois membres de l'UEM, ne disposeront plus des marges de manœuvre laissées aujourd'hui par la politique conjoncturelle. Un choc asymétrique par rapport à la zone euro ne pourra pas être résorbé, ni par un abaissement des taux directeurs des banques centrales nationales, ni par une dévalorisation des monnaies nationales. Quant à la politique budgétaire, elle sera contrainte par les dispositions du Pacte de stabilité et de croissance, et en particulier par le plafond des $3 \%$ de déficit public sur PIB, ce qui limitera le jeu des stabilisateurs fiscaux automatiques. L'adhésion à l'UEM pourrait alors signifier des bouclages d'ajustement aux chocs s'effectuant au détriment de l'emploi.

Ce travail prend pour point de départ la réalisation du critère de Kenen. Ce choix unique nous permet d'approfondir l'approche de cet auteur: nous reformulons le critère originel de diversification sectorielle des exportations, pour transposer ensuite la méthode adoptée à leur diversification géographique. De cette façon, nous pouvons évaluer le risque de choc externe asymétrique, qu'il soit sectoriel - dû à une forte dépendance sur un ou plusieurs produits, ou géographique - dû à une forte dépendance sur un ou plusieurs pays clients. Nous nous intéressons donc aux chocs de demande externe, dont un exemple nous est donné par la crise survenue en Finlande au début de la décennie 90, qui faisait suite à l'effondrement de l'un de ses principaux clients, l'URSS. Barry Eichengreen (1994) prend cet exemple pour souligner l'utilité de la politique de change comme moyen d'ajustement aux chocs asymétriques, les autorités finlandaises ayant laissé se déprécier la markka de près de $30 \%$ en 18 mois (de janvier 1991 à juin 1992).

Dans une première partie, nous construisons deux indicateurs d'exposition aux chocs de demande asymétriques externes, en pondérant deux indicateurs de symétrie (sectorielle et géographique) des ventes extérieures d'un PECO et de l'UEM par le taux d'exportation du pays adhérent. Les deux indicateurs sont ensuite calculés pour la période 1993-2000 à partir 
des données de la base CHELEM ${ }^{1}$, pour les dix PECO qui adhèrent à l’UE en 2004 ou 2007. Nous pouvons ainsi établir deux classements à partir des résultats d'indicateurs d'exposition aux chocs de branche et de destination. Notre objectif est d'extraire les pays les plus exposés, et qui de ce fait pourraient avoir intérêt à reporter leur adhésion à l'union monétaire ou à créer préalablement un fonds de stabilisation conjoncturel. Dans la seconde partie, nous déterminons sur quels produits et quels clients particuliers les PECO les plus exposés risquent de connaître un choc asymétrique vis-à-vis de l'UEM, et nous proposons une mesure de l'impact macroéconomique potentiel de ces chocs relativement au PIB des pays touchés. A titre de comparaison, les mêmes calculs (première et deuxième partie) sont effectués pour la Finlande et l'Irlande, qui sont de toutes les économies de la zone euro les plus vulnérables aux chocs sectoriels et géographiques ${ }^{2}$. Le choix d'une base de données agrégée (CHELEM comprend 71 branches) permet l'étude des chocs sectoriels à impact macroéconomique conséquent. Un découpage trop fin capterait des chocs à effet généralement minime sur le PIB et ne nécessitant pas d'ajustement ultérieur. Dans la troisième partie, nous présentons des éléments sur la convergence réelle avec l’UEM des pays du groupe le plus exposé, et sur l'éventualité d'une réalisation endogène de cette convergence.

Une telle démarche est originale. La symétrie de la géographie commerciale au sein des unions monétaires ne fait pas l'objet d'étude systématique, alors que les asymétries constatées peuvent être très importantes, comme le montre le cas irlandais. La symétrie des structures de production est le plus souvent envisagée sous l'angle de la progression du commerce intrabranche bilatéral, qui, comme nous le verrons, ne témoigne pas nécessairement d'une convergence des structures d'exportation multilatérales, ni d'une moindre vulnérabilité aux chocs asymétriques sectoriels externes. Cela a des implications importantes pour le débat sur le caractère endogène de l'optimalité des unions monétaires. La théorie endogène stipule que l'intensification du commerce intrabranche découlant de l'unification monétaire réduit l'asymétrie des chocs, ce qui contribue à rendre les cycles plus synchrones (J. A. Frankel et K. Rose, 1998). Dans la troisième partie, nous vérifierons que la hausse de l'indicateur de Grubel-Lloyd, observée au cours de la période 1993-2000 pour une majorité de PECO, n'implique pas de diminution obligée de l'indicateur d'exposition aux chocs sectoriels asymétriques externes. La réduction endogène de l'asymétrie des chocs ne s'appliquerait donc pas dans ce cas de figure.

\footnotetext{
${ }^{1}$ Comptes harmonisés sur les échanges et l'économie mondiale, base de données du CEPII (Centre d'études prospectives et d'informations internationales). Les données harmonisées nécessaires à nos calculs ne sont disponibles qu'à partir de 1993.

${ }^{2}$ D’après des calculs de l'auteur à partir de CHELEM.
} 


\title{
I- Elaboration des indicateurs et classement des pays candidats à l'UEM en fonction de leur exposition aux chocs
}

\author{
De l'indicateur de Hirschman à l'indicateur d'exposition aux chocs asymétriques \\ externes
}

C’est au cours des années 60 que s'ébauche la théorie des zones monétaires optimales, chaque auteur avançant un critère d'optimalité spécifique. Dans « The theory of optimum currency area : an eclectic view », P. Kenen (1969) fait écho aux contributions de R. Mundell (1961) et de R. MacKinnon (1963). Son idée est que la diversification de la production d'un pays membre d'un ensemble à monnaie unique, qui se reflète sur celle de ses exportations vers l'ensemble du monde, minimise l'impact macroéconomique d'un choc extérieur. Il survient bien des chocs sectoriels, comme des chocs de demande externe, mais ils n'appellent que des ajustements mineurs. De plus, la présence d'un grand nombre de secteurs fait qu'en moyenne les chocs positifs compensent les chocs négatifs. Dès lors, les économies à structure de production multiforme constituent de bonnes candidates aux unions monétaires, alors que les économies concentrées sur un petit nombre d'activités risquent de fortes perturbations macroéconomiques en cas de choc sectoriel.

\section{Symétrie des structures d'exportation par branche et par destination}

Une application directe de la thèse de Kenen consiste à calculer des indicateurs nationaux de diversification sectorielle multilatérale des exportations, à partir de la méthode de Hirschman $^{3}$. Elle comporte cependant des insuffisances.

La comparaison des indicateurs de Hirschman des PECO et de l'UEM peut être trompeuse. Si certains indicateurs nationaux s'avéraient proches de celui de l'UEM en reflétant une concentration plutôt faible, on pourrait conclure à l'absence de risques de choc asymétrique sectoriel. Mais une telle conclusion serait incorrecte : une même mesure de la diversification pour deux économies peut en effet masquer des asymétries des deux structures d'exportation par branche. Il convient donc de calculer un autre indicateur, cette fois-ci bilatéral, et qui permet d'évaluer, pour chaque PECO, la symétrie de la structure de ses exportations avec celle de l'UEM. L'indicateur de J. M. Finger $(1979)^{4}$ répond à ces attentes. Nous en choisissons une version dérivée dont le mode de calcul figure dans l'encadré 1.

\footnotetext{
3 L’indicateur de Hirschman $(\mathrm{H})$ est égal à la racine carrée de la somme des carrés de la part des exportations totales imputable à chaque produit. Il est compris entre 0 et 1 , de la diversification parfaite à la concentration sur un seul produit.

$$
\mathrm{H}=\left[\sum_{\mathrm{i}=1}\left(\mathrm{x}_{\mathrm{i}}\right)^{2}\right]^{1 / 2} \text {, n étant le nombre de produits d'exportation et } \mathrm{x}_{\mathrm{i}} \text { la part des exportations du produit i dans }
$$
les exportations totales.

${ }^{4}$ J. M. Finger utilise l'indicateur de symétrie des exportations pour montrer que les pays semi-industrialisés sont les principaux bénéficiaires du Système généralisé de préférences (SGP). Au sein des pays en développement, ils détiennent les structures d'exportation vers le Nord les plus proches de celles des pays industrialisés. A la faveur des préférences tarifaires, ils sont donc les mieux placés pour augmenter leurs exportations vers les marchés des pays développés en suscitant des détournements de commerce en défaveur du Nord.
} 


\section{Encadré 1 L’indicateur de symétrie des branches exportatrices}

L'indicateur $\mathrm{S}_{1}$ de symétrie des branches exportatrices de deux pays $\mathrm{A}$ et $\mathrm{B}$, ou d'un pays A vis-à-vis du groupe de ses partenaires B (soit un PECO et l'UEM dans le cas étudié), est calculé comme suit.

Soit $\mathrm{A}_{\mathrm{i}}=\mathrm{X}_{\mathrm{ai}} / \mathrm{X}_{\mathrm{a}}$, , les ventes du pays $\mathrm{A}$ en produits de la branche $\mathrm{i}$ sur ses exportations totales.

Soit $\mathrm{B}_{\mathrm{i}}=\mathrm{X}_{\mathrm{bi}} / \mathrm{X}_{\mathrm{b}}$, les ventes du pays ou de la zone $\mathrm{B}$ en produits de la branche i sur ses exportations totales.

$$
S_{1}=1 / 2 \sum_{i=1}^{n}\left|A_{i}-B_{i}\right|
$$

$\mathrm{S}_{1}$ varie de 0 à 1 . Si $\mathrm{A}$ et $\mathrm{B}$ exportaient la même part pour chaque branche $\left(\mathrm{A}_{\mathrm{i}}=\mathrm{B}_{\mathrm{i}}\right.$ quel que soit i), la somme des valeurs absolues et donc $S_{1}$ seraient égaux à 0 (symétrie parfaite et absence de chocs asymétriques de branche). Si les exportations de A (ou B) étaient systématiquement issues d'une branche ne comptant aucun produit exporté par B (ou A), la somme des valeurs absolues serait égale à 2 et $\mathrm{S}_{1}$ à 1 (dissymétrie parfaite et forte exposition aux chocs).

L'indicateur $\mathrm{S}_{1}$ est conforme à l'approche de J. M. Finger. L'indicateur de Finger est égal à la somme des minimums pour $\mathrm{A}$ et $\mathrm{B}$ des parts de chaque branche dans les exportations totales, ce qui correspond à $\left(1-\mathrm{S}_{1}\right)$. Une valeur de 0 indique alors une dissymétrie parfaite et une valeur de 1 une symétrie parfaite.

L'analyse de Kenen ne repose que sur la seule diversification des branches exportatrices. Elle est pourtant aisément transposable à la diversification des marchés extérieurs. De la même manière qu'une économie fortement exposée sur un petit nombre de branches risque de fortes perturbations macroéconomiques, un pays dont les partenaires commerciaux sont peu nombreux est vulnérable à une récession survenant chez l'un d'entre eux. Plus la concentration géographique des exportations est importante, et plus la chute de la demande d'un partenaire est pénalisante. A l'image de la diversification de branche, la diversification géographique des exportations minimise l'ajustement macroéconomique requis par ce type de choc. De même, elle permet à long terme une compensation des chocs négatifs par les chocs positifs.

La méthode de Hirschman pourrait être utilisée pour calculer un indicateur de diversification multilatérale des marchés extérieurs, mais avec les inconvénients soulignés précédemment. Un même résultat d'indicateur pour un PECO et la zone euro ne signifierait pas nécessairement une liste identique de destinataires, ni un pourcentage équivalent de ventes affecté à chaque partenaire. Nous utiliserons donc un indicateur mesurant la symétrie de la structure des exportations par destination d'un PECO et de l’UEM (voir l'encadré 2). 


\section{Encadré 2 L’indicateur de symétrie des destinations exportatrices}

L'indicateur $S_{2}$ de symétrie géographique des exportations de deux pays $A$ et $B$, ou d'un pays A vis-à-vis du groupe de ses partenaires B (en l'occurrence un PECO et l'UEM), est calculé selon la même logique que l'indicateur $S_{1}$ de symétrie des branches exportatrices.

Soit $A_{j}=X_{a j} / X_{a}$, les exportations du pays $A$ vers le pays $J$ sur ses exportations totales (hors exportations vers le ou les partenaires).

Soit $\mathrm{B}_{\mathrm{j}}=\mathrm{X}_{\mathrm{bj}} / \mathrm{X}_{\mathrm{b}}$. , les exportations du pays ou de la zone $\mathrm{B}$ vers le pays $\mathrm{J}$ sur ses exportations totales (hors exportations vers le partenaire).

$$
S_{2}=1 / 2 \sum_{j=1}^{n}|A j-B j|
$$

$\mathrm{S}_{2}$ varie de 0 à 1 . Si $\mathrm{A}$ et $\mathrm{B}$ exportaient la même part vers chaque destinataire du reste du monde $\left(A_{j}=B_{j}\right.$ quel que soit $\left.\mathrm{j}\right)$, la somme des valeurs absolues et donc $S_{2}$ seraient égaux à 0 (symétrie parfaite et absence de chocs asymétriques géographiques). Si A (ou B) exportait systématiquement vers une destination vers laquelle B (ou A) n'exportait pas, la somme des valeurs absolues serait égale à 2 et $\mathrm{S}_{2}$ à 1 (dissymétrie parfaite et forte exposition aux chocs).

\section{La prise en compte du taux d'exportation}

Les indicateurs de symétrie sectorielle et géographique des exportations permettent une mesure plus fiable des asymétries entre partenaires que la comparaison des indicateurs de Hirschman. Mais si l'on souhaite analyser le risque de choc asymétrique externe d'un PECO vis-à-vis de l'UEM, il est nécessaire d'introduire son taux d'exportation dans le raisonnement ${ }^{5}$. En effet, l'impact macroéconomique d'un choc externe dépend tout autant de l'asymétrie de la répartition des ventes extérieures que du rapport exportations / PIB de l'économie touchée. Une asymétrie élevée sera peu préoccupante si elle se combine avec un faible taux d'exportation, mais elle le sera doublement dans le cas contraire. Nous construisons donc deux indicateurs d'exposition aux chocs asymétriques externes, sectoriel et géographique, en pondérant les deux indicateurs de symétrie précédents par le taux d'exportation du pays candidat à l’UEM (cf. l'encadré 3).

\footnotetext{
${ }^{5}$ On pourrait considérer le différentiel de taux d'exportation entre un PECO et l'UEM. Mais notre objectif est d'établir un classement des dix pays concernés, et celui-ci n'est pas affecté par la seule prise en compte du taux d'exportation du PECO.
} 


\section{Encadré 3 Les indicateurs d'exposition aux chocs asymétriques externes}

Les deux indicateurs précédents (encadrés 1 et 2) donnent la synthèse des asymétries existant entre les structures d'exportation, par branche et par destination, d'un PECO et de l'UEM. Pour évaluer le risque de choc asymétrique externe, il est nécessaire de pondérer ces indicateurs par le taux d'exportation du pays candidat à l'UEM, puisque l'impact macroéconomique d'un choc sectoriel ou géographique est d'autant plus important que le taux d'exportation est élevé.

En prenant le taux d'exportation en pourcentage, nous obtenons les indicateurs $E_{1}$ d'exposition aux chocs externes sectoriels et $E_{2}$ d'exposition aux chocs externes géographiques :

$$
\begin{aligned}
& \mathrm{E}_{1}=\mathrm{S}_{1} \cdot\left(\left(\mathrm{X}_{\mathrm{cm}} / \mathrm{PIB}_{\mathrm{c}}\right) \cdot 100\right) \\
& \mathrm{E}_{2}=\mathrm{S}_{2} \cdot\left(\left(\mathrm{X}_{\mathrm{c}(\mathrm{m}-\mathrm{z})} / \mathrm{PIB}_{\mathrm{c}}\right) \cdot 100\right)
\end{aligned}
$$

Où $\mathrm{PIB}_{\mathrm{c}}$ est le PIB du pays candidat à l’UEM.

Et où $X_{\mathrm{cm}}$ et $\mathrm{X}_{\mathrm{c}(\mathrm{m}-\mathrm{z})}$ sont respectivement les exportations du pays candidat vers l'ensemble du monde et vers l'ensemble du monde hormis la zone euro.

La prise en compte des seules exportations extra-UEM pour le calcul de $E_{2}$ se justifie de la façon suivante : lorsqu'un ou plusieurs pays membres de l'UEM connaît une récession brutale ayant pour conséquence un tassement des exportations de leurs partenaires, le choc asymétrique au sein de l'union monétaire touche les premiers et non les seconds, de même que la nécessité de l'ajustement incombe aux premiers vis-à-vis des seconds. Il convient donc d'exclure les ventes des pays candidats vers l'UEM pour apprécier leur risque de chocs asymétriques géographiques vis-à-vis de cette zone.

Si l'on admet des bornes théoriques allant de 0 à $100 \%$ pour le taux d'exportation, avec des indicateurs $S_{1}$ et $S_{2}$ variant dans l'intervalle $[0,1]$, les indicateurs d'exposition aux chocs varient au sein de l'intervalle $[0,100]$ (de la plus petite exposition à la plus forte exposition).

\section{Une exposition aux chocs souvent prononcée}

Les indicateurs d'exposition aux chocs asymétriques externes dépendent de la convergence des structures d'exportation de l'UEM et des pays candidats, ainsi que de l'ouverture commerciale de ces derniers. Ces deux facteurs sont largement influencés par une série d'accords coïncidant avec la déliquescence du système de planification centralisé et avec le démantèlement du CAEM, qui se traduit par une chute du commerce des PECO avec l'exURSS. Ces accords contribuent à libéraliser le commerce extérieur de ces pays et à développer leurs échanges, en particulier avec l’Union européenne : accords de coopération économique et commerciale de première génération conclus à partir de la fin de la décennie 80, octroi par l’Union de la clause de la nation la plus favorisée en matière tarifaire, extension du mécanisme communautaire du SGP (Système généralisé de préférences) dans le cadre du programme PHARE, et accords de seconde génération signés pour la plupart sur la période 1991-93. Ces derniers, qualifiés également d' «accords européens », mettent en oeuvre sur une période de 10 ans des zones de libre-échange bilatérales pour les produits industriels, entre l'Union et chacun de ses futurs membres. 
Les conditions sont donc réunies pour une rénovation des tissus industriels et une redéfinition des spécialisations sous l'impulsion des firmes ouest-européennes, qui prennent une part active aux programmes de privatisation, créent des joint ventures avec des entreprises locales, ou les intègrent dans leur réseau commercial par transfert de technologie. Au cours de la décennie 90, les secteurs d'exportation vers l'Union européenne les plus dynamiques sont ceux pour lesquels la présence étrangère est particulièrement importante (M. Freudenberg et F. Lemoine, 1999). On devrait donc observer, à moyen et long terme, un mouvement d'ouverture commerciale des pays en transition s'accompagnant d'une convergence de leurs structures de production avec l'Union.

Si le mouvement d'ouverture commerciale se confirme de la période 1993-1996 à la période 1997-2000, il n'en est pas de même de la convergence des structures de production avec l'UEM, lorsqu'on l'envisage, comme c'est le cas ici, sous l'angle de la structure des exportations par branche. A l'exception de la Pologne, les indicateurs de symétrie des exportations par branche stagnent ou connaissent une légère détérioration d'une période à l'autre (voir la $1^{\text {ère }}$ colonne du tableau 1). On peut s'étonner d'un tel résultat, puisque l'intégration des économies de l'Est au sein des réseaux des firmes multinationales européennes laisse supposer une augmentation de leurs échanges intrabranches avec l'UEM, qui pourrait témoigner d'un certain rapprochement des structures d'exportations multilatérales. Six PECO connaissent cette progression du commerce intrabranche de la période 1993-1996 à la période $1997-2000^{6}$ (par ordre de progression absolue de l’indicateur intrabranche, il s'agit de la Roumanie, de la Slovénie, de la République tchèque, de la Lettonie, de la Pologne, et de la Lituanie ${ }^{7}$ ). Mais la contradiction n'est qu'apparente, puisque les deux indicateurs obéissent à une logique différente, bilatérale dans le cas des échanges intrabranches, et multilatérale pour la symétrie des exportations par branche. Elle montre simplement que la convergence des structures d'exportation bilatérales n'a pas nécessairement de prolongement pour les structures d'exportation multilatérales.

\footnotetext{
${ }^{6}$ En considérant la moyenne annuelle de la période 1997-2000 par rapport à la moyenne annuelle de la période 1993-1996. Calculs de l'auteur à partir d'un indicateur de Grubel et Lloyd - corrigé du déséquilibre commercial et de la décomposition en 71 branches de CHELEM. L'ensemble des résultats et la méthode de calcul de l'indicateur figurent dans le tableau 5 et en annexe.

${ }^{7}$ En considérant l'évolution du ratio de Grubel-Lloyd corrigé du déséquilibre commercial de l'année 1993 à l'année 2000, sept pays de l'Est sur dix connaissent une progression de la part des échanges intrabranches avec l’UEM, la Slovaquie venant s'ajouter à la liste des six pays cités.
} 


\section{Tableau 1 Indicateurs d'exposition aux chocs asymétriques sectoriels}

\begin{tabular}{|c|c|c|c|c|c|c|}
\hline $\begin{array}{c}\text { Classement } \\
\text { des PECO } \\
(\mathbf{1})\end{array}$ & $\begin{array}{c}\text { Indicateur de symétrie } \\
\text { des exportations par } \\
\text { branche }\end{array}$ & \multicolumn{2}{c|}{$\begin{array}{c}\text { Taux d'exportation } \\
\text { multilatéral (2) }\end{array}$} & \multicolumn{2}{c|}{$\begin{array}{c}\text { Indicateur d'exposition } \\
\text { aux chocs sectoriels (3) }\end{array}$} \\
\cline { 2 - 7 } & $\begin{array}{c}\text { Moyenne } \\
\mathbf{1 9 9 3 - 1 9 9 6}\end{array}$ & $\begin{array}{c}\text { Moyenne } \\
\mathbf{1 9 9 7 - 2 0 0 0}\end{array}$ & $\begin{array}{c}\text { Moyenne } \\
\mathbf{1 9 9 3 - 1 9 9 6}\end{array}$ & $\begin{array}{c}\text { Moyenne } \\
\mathbf{1 9 9 7 - 2 0 0 0}\end{array}$ & $\begin{array}{c}\text { Moyenne } \\
\mathbf{1 9 9 3 - 1 9 9 6}\end{array}$ & $\begin{array}{c}\text { Moyenne } \\
\mathbf{1 9 9 7 - 2 0 0 0}\end{array}$ \\
\hline Pologne & 0,40 & 0,36 & 16 & 17 & 6,6 & 6,2 \\
\hline Tchéquie & 0,28 & 0,28 & 35 & 47 & 9,7 & 13,1 \\
\hline Roumanie & 0,49 & 0,54 & 21 & 25 & 10,5 & 13,4 \\
\hline Slovénie & 0,32 & 0,32 & 44 & 44 & 14,1 & 14,3 \\
\hline Lituanie & 0,49 & 0,51 & 38 & 33 & 18,5 & 16,6 \\
\hline Slovaquie & 0,32 & 0,33 & 44 & 51 & 14,3 & 16,7 \\
\hline Hongrie & 0,30 & 0,34 & 29 & 50 & 8,6 & 17,0 \\
\hline Bulgarie & 0,44 & 0,47 & 40 & 42 & 17,8 & 19,6 \\
\hline Lettonie & 0,60 & 0,63 & 41 & 45 & 24,7 & 28,5 \\
\hline Estonie & 0,48 & 0,52 & 36 & 70 & 17,3 & 36,5 \\
\hline
\end{tabular}

(1) Du pays le moins exposé au pays le plus exposé, à partir du résultat de la moyenne annuelle de la période la plus récente (1997-2000).

(2) Exportations vers l'ensemble du monde sur PIB, en pourcentage.

(3) L'indicateur d'exposition aux chocs, dont la construction est expliquée dans l'encadré 3, est borné par les valeurs 0 et 100 . Il est égal au produit de l'indicateur de symétrie des exportations par branche (cf. encadré 1) par le taux d'exportation multilatéral exprimé en pourcentage. Les résultats de la colonne donnent par pays la moyenne des indicateurs annuels pour les deux périodes. Ils diffèrent donc légèrement de ceux qui résulteraient du produit des valeurs des $1^{\text {ère }}$ et $3^{\text {ème }}$ colonnes et du produit des valeurs des $2^{\text {ème }}$ et $4^{\text {ème }}$ colonnes (soit le produit des moyennes pour les deux périodes).

En règle générale, sans amélioration des indicateurs de symétrie et avec une ouverture commerciale qui s'intensifie, l'exposition aux chocs sectoriels s'accentue d'une période à l'autre (cf. tableau 1). Les niveaux atteints indiquent de fortes disparités. Ils peuvent être comparés à la situation des deux pays membres de l'UEM dont la structure d'exportation est la plus concentrée, soit la Finlande et l'Irlande (cf. tableau 3). Une majorité de PECO ont une exposition prononcée, puisqu'elle est similaire ou supérieure à celle de la Finlande, tout en restant inférieure à celle de l’Irlande.

Pays baltes et Slovaquie : une forte sensibilité aux chocs géographiques

Contrairement à la symétrie des exportations par branche, la symétrie des exportations par destination connaît une amélioration générale d'une période à l'autre, Pologne exclue (cf. tableau 2). Les taux d'exportation extra-UEM n'enregistrent pas nécessairement de progression. Dans la plupart des cas, l'exposition aux chocs ne connaît pas de changement significatif. Mais les niveaux atteints reflètent à nouveau des écarts importants. De plus, une majorité de pays ont une forte exposition, supérieure à celle de la Finlande, voire très supérieure dans le cas de la Slovaquie et des trois pays baltes. Ces quatre pays ont aussi une exposition proche ou plus élevée que celle de l’Irlande. Ils figuraient déjà parmi les moins bien classés pour la sensibilité aux chocs sectoriels. Nous y reviendrons en seconde partie lorsque nous détaillerons les branches et les destinations concernées par les risques de chocs à fort impact macroéconomique. 
Tableau 2 Indicateurs d'exposition aux chocs asymétriques géographiques

\begin{tabular}{|c|c|c|c|c|c|c|}
\hline $\begin{array}{c}\text { Classement des } \\
\text { PECO (1) }\end{array}$ & $\begin{array}{c}\text { Indicateur de symétrie } \\
\text { des exportations par } \\
\text { destination }\end{array}$ & \multicolumn{2}{|c|}{$\begin{array}{c}\text { Taux d'exportation } \\
\text { extra-UEM (2) }\end{array}$} & \multicolumn{2}{c|}{$\begin{array}{c}\text { Indicateur d'exposition } \\
\text { aux chocs géographiques } \\
\text { (3) }\end{array}$} \\
\cline { 2 - 7 } & $\begin{array}{c}\text { Moyenne } \\
\mathbf{1 9 9 3 - 1 9 9 6}\end{array}$ & $\begin{array}{c}\text { Moyenne } \\
\mathbf{1 9 9 7 - 2 0 0 0}\end{array}$ & $\begin{array}{c}\text { Moyenne } \\
\mathbf{1 9 9 3 - 1 9 9 6}\end{array}$ & $\begin{array}{c}\text { Moyenne } \\
\mathbf{1 9 9 7 - 2 0 0 0}\end{array}$ & $\begin{array}{c}\text { Moyenne } \\
\mathbf{1 9 9 3 - 1 9 9 6}\end{array}$ & $\begin{array}{c}\text { Moyenne } \\
\mathbf{1 9 9 7 - 2 0 0 0}\end{array}$ \\
\hline Bulgarie & 0,54 & 0,53 & 6 & 5 & 3,3 & 2,7 \\
\hline Pologne & 0,41 & 0,46 & 7 & 7 & 2,8 & 3,3 \\
\hline Roumanie & 0,50 & 0,40 & 12 & 11 & 5,8 & 4,3 \\
\hline Hongrie & 0,43 & 0,36 & 11 & 18 & 4,8 & 6,4 \\
\hline Tchéquie & 0,56 & 0,49 & 17 & 19 & 9,3 & 9,3 \\
\hline Slovénie & 0,56 & 0,55 & 16 & 17 & 9,1 & 9,3 \\
\hline Lituanie & 0,63 & 0,60 & 26 & 22 & 16,7 & 13,2 \\
\hline Slovaquie & 0,76 & 0,68 & 27 & 24 & 20,5 & 16,1 \\
\hline Lettonie & 0,58 & 0,51 & 27 & 32 & 15,5 & 16,3 \\
\hline Estonie & 0,64 & 0,57 & 23 & 44 & 14,9 & 24,8 \\
\hline
\end{tabular}

(1) Du pays le moins exposé au pays le plus exposé, à partir du résultat de la moyenne annuelle de la période la plus récente (1997-2000).

(2) Exportations vers l'ensemble du monde zone euro exclue, sur PIB, en pourcentage.

(3) L’indicateur d'exposition aux chocs, dont la construction est expliquée dans l'encadré 3, est borné par les valeurs 0 et 100 . Il est égal au produit de l’indicateur de symétrie des exportations par destination (cf. encadré 2) par le taux d'exportation extra-UEM exprimé en pourcentage. Les résultats de la colonne donnent par pays la moyenne des indicateurs annuels pour les deux périodes. Ils diffèrent donc légèrement de ceux qui résulteraient du produit des valeurs des $1^{\text {ère }}$ et $3^{\text {ème }}$ colonnes et du produit des valeurs des $2^{\text {ème }}$ et $4^{\text {ème }}$ colonnes (soit le produit des moyennes pour les deux périodes).

\section{Tableau 3 Indicateurs d'exposition aux chocs sectoriel et géographique de la Finlande et de l'Irlande}

\begin{tabular}{|c|c|c|c|c|c|c|}
\cline { 2 - 7 } \multicolumn{1}{c|}{} & \multicolumn{2}{c|}{$\begin{array}{c}\text { Indicateur de symétrie des } \\
\text { exportations par branche }\end{array}$} & \multicolumn{2}{c|}{$\begin{array}{c}\text { Taux d'exportation } \\
\text { multilatéral (1) }\end{array}$} & $\begin{array}{c}\text { Indicateur d'exposition aux } \\
\text { chocs sectoriels (2) }\end{array}$ \\
\cline { 2 - 7 } & $\begin{array}{c}\text { Moyenne } \\
\mathbf{1 9 9 3 - 1 9 9 6}\end{array}$ & $\begin{array}{c}\text { Moyenne } \\
\mathbf{1 9 9 7 - 2 0 0 0}\end{array}$ & $\begin{array}{c}\text { Moyenne } \\
\mathbf{1 9 9 3 - 1 9 9 6}\end{array}$ & $\begin{array}{c}\text { Moyenne } \\
\mathbf{1 9 9 7 - 2 0 0 0}\end{array}$ & $\begin{array}{c}\text { Moyenne } \\
\mathbf{1 9 9 3 - 1 9 9 6}\end{array}$ & $\begin{array}{c}\text { Moyenne } \\
\mathbf{1 9 9 7 - 2 0 0 0}\end{array}$ \\
\hline FINLANDE & 0,45 & 0,48 & 30 & 34 & 13,5 & 16,3 \\
\hline IRLANDE & 0,52 & 0,54 & 60 & 74 & 31,7 & 40,1 \\
\hline & \multicolumn{2}{|c|}{$\begin{array}{c}\text { Indicateur de symétrie des } \\
\text { exportations par destination }\end{array}$} & $\begin{array}{c}\text { Taux d'exportation } \\
\text { extra-UEM (3) }\end{array}$ & $\begin{array}{c}\text { Indicateur d'exposition aux } \\
\text { chocs géographiques (4) }\end{array}$ \\
\hline & $\begin{array}{c}\text { Moyenne } \\
\mathbf{1 9 9 3 - 1 9 9 6}\end{array}$ & $\begin{array}{c}\text { Moyenne } \\
\mathbf{1 9 9 7 - 2 0 0 0}\end{array}$ & $\begin{array}{c}\text { Moyenne } \\
\mathbf{1 9 9 3 - 1 9 9 6}\end{array}$ & $\begin{array}{c}\text { Moyenne } \\
\mathbf{1 9 9 7 - 2 0 0 0}\end{array}$ & $\begin{array}{c}\text { Moyenne } \\
\mathbf{1 9 9 3 - 1 9 9 6}\end{array}$ & $\begin{array}{c}\text { Moyenne } \\
\mathbf{1 9 9 7 - 2 0 0 0}\end{array}$ \\
\hline FINLANDE & 0,30 & 0,31 & 20 & 23 & 6,0 & 7,1 \\
\hline IRLANDE & 0,36 & 0,34 & 36 & 45 & 13,1 & 15,6 \\
\hline
\end{tabular}

(1) Exportations vers l'ensemble du monde, sur PIB, en \%.

(2) Voir la note (3) du tableau 1.

(3) Exportations vers l'ensemble du monde zone euro exclue, sur PIB, en \%.

(4) Voir la note (3) du tableau 2. 


\section{II- La mesure de l'impact potentiel des chocs asymétriques : quels sont les risques majeurs?}

Les deux indicateurs d'exposition aux chocs utilisés dans la partie précédente prennent en compte l'ensemble des asymétries existant entre un PECO et l'UEM, quelle que soit leur ampleur. Leur caractère synthétique ne permet pas une analyse détaillée, branche par branche, ou destination par destination. Nous nous proposons maintenant d'isoler les branches et destinations pour lesquelles l'impact macroéconomique potentiel d'un choc asymétrique externe est significatif, en précisant d'abord comment mesurer l'impact maximal d'un tel choc.

\section{La mesure de l’impact macroéconomique maximal d'un choc sectoriel ou géographique}

Pour mesurer l'impact macroéconomique d'un choc asymétrique externe, supposons le cas extrême d'un choc entraînant d'une année sur l'autre l'arrêt total des exportations d'un pays candidat à l'UEM et de la zone euro, pour une branche ou une destination donnée. Dans ce cas, chacun des deux partenaires subit un préjudice équivalant au rapport des ventes extérieures correspondantes à son PIB (en pourcentage). Considérons un choc touchant le pays candidat. Le degré d'asymétrie du choc est alors mesurable par la différence entre les rapports du pays candidat et de l'UEM. Cette différence représente en points de PIB l'effort d'ajustement macroéconomique que doit fournir ce pays vis-à-vis de la zone euro. Compte tenu de notre supposition initiale, elle mesure l'impact maximal d'un choc (voir l'encadré 4).

\section{Encadré 4 Impact maximal d'un choc asymétrique sectoriel ou géographique}

Les structures d'exportation différenciées par destination et par branche entre chaque pays candidat à l'UEM et la zone euro peuvent être à l'origine de chocs asymétriques. Nous nous proposons d'évaluer leur impact potentiel. La formule suivante permet de mesurer, en pourcentage du PIB, l'impact maximal (IM) d'un choc sectoriel ou géographique pour une année donnée :

$\mathrm{IM}_{\mathrm{czi}}=\left(\left(\mathrm{X}_{\mathrm{ci}} / \mathrm{PIB}_{\mathrm{c}}\right)-\left(\mathrm{X}_{\mathrm{zi}} / \mathrm{PIB}_{\mathrm{z}}\right)\right) \cdot 100$

Où :

- i représente la branche ou la destination qui occasionne une chute des exportations.

- $\mathrm{X}_{\mathrm{ci}}$ et $\mathrm{X}_{\mathrm{zi}}$ correspondent respectivement aux exportations du pays candidat et de la zone euro pour la branche ou la destination $\mathrm{i}$.

- $\mathrm{PIB}_{\mathrm{c}}$ et $\mathrm{PIB}_{\mathrm{z}}$ sont les produits intérieurs bruts respectifs du pays candidat et de la zone euro.

IM $_{\text {czi }}$ est l'impact maximal d'un choc asymétrique relatif à la branche ou la destination i. Comme on considère les chocs touchant un pays candidat à l'UEM par rapport à la zone euro, on obtient un résultat en points de PIB du pays candidat, qui représente l'effort maximal d'ajustement à fournir en cas de choc relatif à la branche ou la destination i. Les chocs à impact les plus élevés sont recensés dans les graphiques 1 et 2 .

Le calcul de l'impact maximal d'un choc repose sur l'hypothèse d'un arrêt complet des exportations par branche ou destination, d'une année sur l'autre. Les résultats obtenus ne sont donc que des plafonds théoriques, l'impact d'un choc dépendant de la proportion des exportations touchées, et cette proportion n'atteignant jamais $100 \%$. 


\section{L'Estonie et la Lettonie concentrent les risques majeurs}

Lorsque l'on recense les couples pays/branche (graphique 1) et pays/destination (graphique 2) susceptibles d'être à l'origine des chocs à fort impact macroéconomique, l'Estonie et la Lettonie sont largement représentées. En règle générale, ces chocs seraient plus intenses que ceux qui toucheraient la Finlande mais moins intenses que ceux qui pourraient atteindre l'Irlande (cf. graphique 3).

\section{Les chocs concerneraient plutôt des branches traditionnelles}

Une majorité de branches traditionnelles à faible intensité technologique sont concernées par les chocs sectoriels, comme la confection pour la Bulgarie et la Roumanie, la métallurgie non ferreuse pour la Bulgarie, ou le fer-acier pour la Slovaquie. Les produits raffinés du pétrole représentent un risque pour les trois pays baltes, particulièrement important pour l'Estonie et la Lettonie. Seules deux branches liées aux nouvelles technologies figurent parmi les risques potentiels les plus élevés, le matériel de télécommunication pour l’Estonie et le matériel informatique pour la Hongrie. Le dynamisme des exportations estoniennes de téléphones et d'équipements de télécommunication s'explique largement par les investissements directs étrangers, notamment finlandais, qui exploitent des différences de coûts salariaux et d'imposition des bénéfices. Ce sont aussi les investissements étrangers, qui ont connu une très forte croissance vers la Hongrie dans le secteur électronique, qui expliquent les chocs potentiels sur les produits informatiques. Cette situation rappelle celle de l'Irlande qui a connu une grande vigueur des IDE électroniques à la faveur de son intégration au marché communautaire, et pour qui l'intensité d'un choc sur le matériel informatique serait trois plus importante que pour la Hongrie (cf. graphique 3). La concentration sur des branches à technologie avancée est préférable à une spécialisation portant sur des branches traditionnelles, les premières profitant d'un taux de croissance plus élevée de la demande mondiale. Elle ne met cependant pas à l'abri des chocs sectoriels de délocalisation vers de nouveaux pays d'accueil. Ce risque existe aussi pour l'Irlande, dont la dynamique de croissance au sein d'un ensemble à monnaie unique se traduit par une appréciation du taux de change réel qui pénalise la compétitivité des filiales étrangères. 


\section{Graphique 1 Les chocs asymétriques potentiels les plus importants Pays candidat à l'UEM / Branche majeure en crise}

En \% du PIB du pays candidat à l'UEM, moyenne annuelle 1996-2000

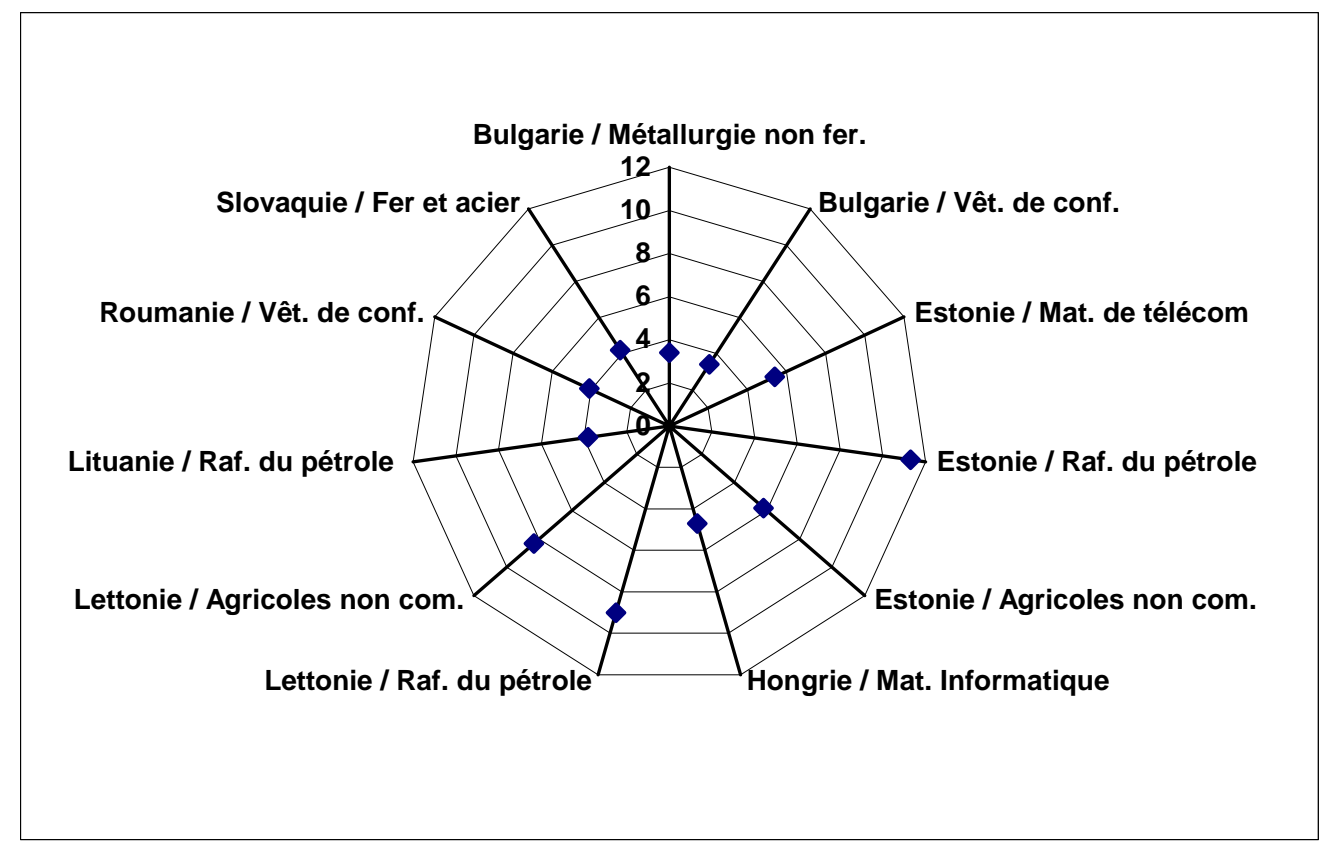

Note: les résultats du graphique présentent en points de PIB la mesure de l'impact maximal (cf. encadré 4) que subirait un pays candidat à l'UEM en cas de crise touchant l'une de ses branches d'exportation dominantes. N'ont été retenus que les impacts annuels moyens supérieurs à 3 points de PIB sur la période 1996-2000, avec un minimum de 2 points de PIB par an (à l'exception de Hongrie/Matériel informatique en 1996 et de Estonie/Matériel de télécommunication en 1996 et 1997).

\section{Les destinations critiques concernent essentiellement des futurs membres de l'UEM}

A l'exception de la dépendance de l'Estonie à l'égard de la Russie, d'ailleurs en nette diminution au cours de la décennie 90 du fait d'une réorientation des échanges avec l'Ouest ${ }^{8}$, les destinations impliquant d'éventuels chocs asymétriques concernent uniquement des pays qui, à terme, devraient adhérer à l'UEM. Les risques les plus importants reflètent le maintien d'intenses courants d'échanges entre la Slovaquie et la Tchéquie, ainsi que les liens commerciaux entretenus entre l'Estonie et la Suède, dont les filiales des firmes multinationales alimentent largement les flux d'échange bilatéraux.

\footnotetext{
${ }^{8}$ La part des exportations de l'Estonie vers la Russie dans ses exportations totales diminue progressivement, de $14 \%$ en 1993 à $3 \%$ en 2000 (contre $1 \%$ en 2000 pour l'UEM). Il est d'ailleurs difficile de distinguer, dans la chute des exportations de l'Estonie vers la Russie de 1997 à 1998, ce qui relève du mouvement de long terme de réorientation des échanges de ce qui relève de la forte baisse du PIB russe, de près de $5 \%$ en 1998 . La chute des exportations estoniennes vers la Russie en 1998 est plus que compensée par une augmentation de ses exportations totales.
} 
Graphique 2 Les chocs asymétriques potentiels les plus importants : Pays candidat à l'UEM / Partenaire majeur en crise

En \% du PIB du pays candidat à l’UEM, moyenne annuelle 1996-2000

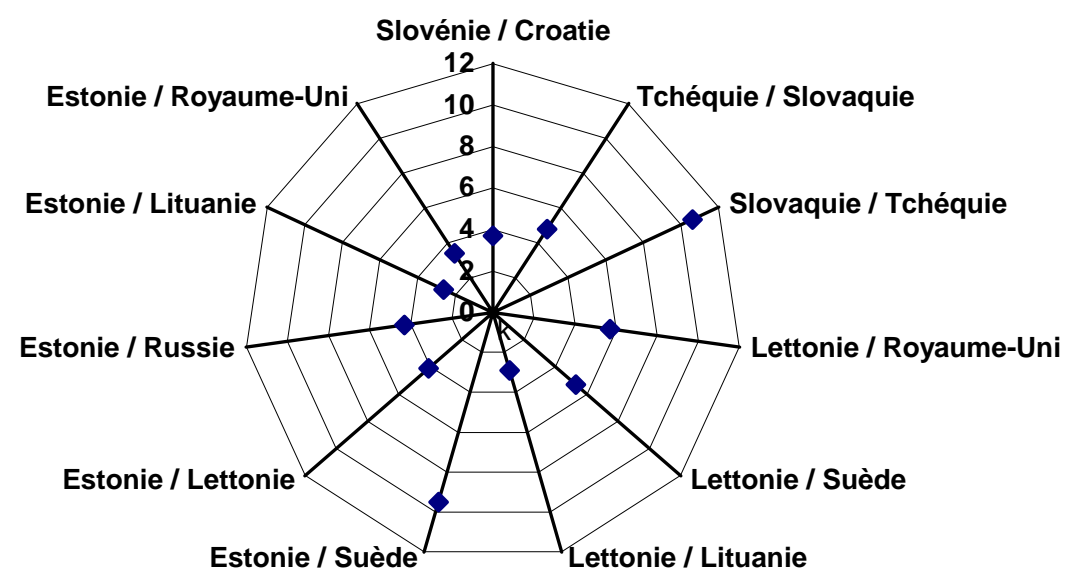

Note: les résultats du graphique présentent en points de PIB la mesure de l'impact maximal (cf. encadré 4) que subirait un pays candidat à l'UEM en cas de crise chez un partenaire commercial majeur. N'ont été retenus que les impacts annuels moyens supérieurs à 2,5 points de PIB sur la période 1996-2000, avec un minimum de 2 points de PIB par an.

\section{Graphique 3 Les chocs sectoriels et géographiques potentiels les plus intenses de la Finlande et de l'Irlande}

En \% du PIB du pays exposé, moyenne annuelle 1996-2000

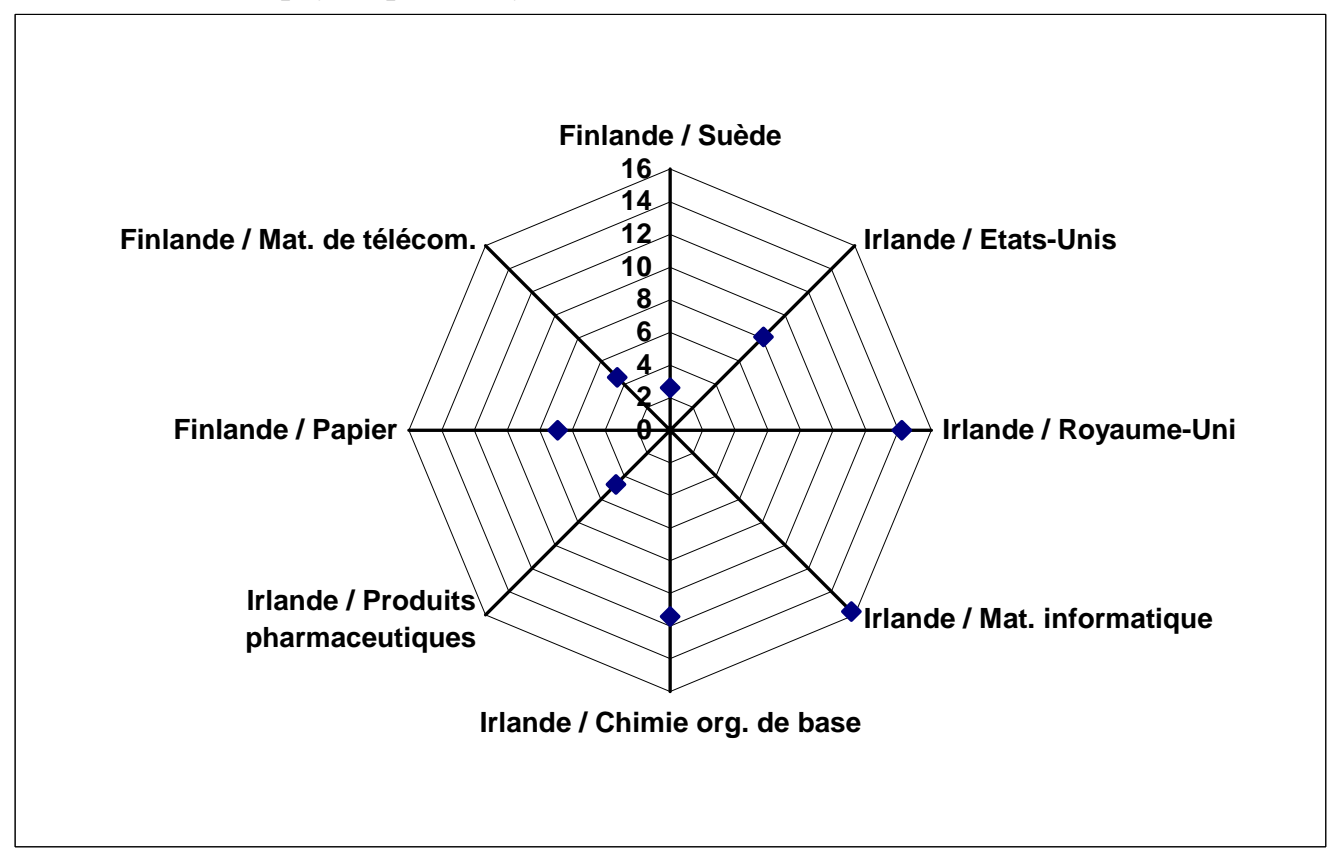

Note : pour le graphique 3, la méthode du graphique 1 a été appliquée pour les chocs sectoriels et celle du graphique 2 pour les chocs géographiques. 
Les pays candidats les plus sensibles aux chocs géographiques (soit ceux figurant sur le graphique 2) font tous partie de la première vague d'élargissement de 2004. Il est possible que leur prochaine participation au marché unique contribue à diversifier leurs partenaires commerciaux européens, en aplanissant le risque de choc. Dans le cas contraire, et dans l'hypothèse d'adhésion à l'UEM de tous les pays membres actuels et futurs de l'Union européenne, le problème se posera en terme de choc asymétrique géographique dérivé. Le foyer de la crise se situera déjà au sein de l'UEM, et il incombera en priorité au pays touché de s'ajuster par rapport à ses partenaires de la zone euro. L'ampleur de l'ajustement incombant au pays subissant un choc dérivé, soit une chute des exportations vers le partenaire atteint par le choc initial, sera nécessairement moindre.

Des chocs dérivés pourront aussi intervenir en cas de crise dans des pays membres actuels de l'UEM. Ni la méthode de calcul des indicateurs d'exposition aux chocs asymétriques géographiques (cf. encadré 3), ni la recherche des chocs potentiels les plus importants (cf. graphique 2), n'en tenait compte. Lorsque l'on calcule l'indicateur d'exposition avec le taux d'exportation multilatéral et non plus avec le taux d'exportation extra-UEM, le classement des pays candidats du tableau 2, qui concerne la période 1997-2000, reste relativement stable (cf. tableau 4). Il se détériore toutefois sensiblement pour la Bulgarie, qui passe de la première à la cinquième place, et légèrement pour la Slovénie et la Tchéquie, qui régressent chacune de deux places. Cela est dû à une forte exposition sur les marchés grecque (Bulgarie), italien (Bulgarie et Slovénie) et allemand (Tchéquie). Par contrecoup, la Lettonie et la Lituanie, dont les exportations en dehors de la zone euro sont beaucoup plus élevées que vers l'UEM, améliorent leur classement de trois places.

\section{Tableau 4 Indicateurs d'exposition aux chocs asymétriques géographiques - Classement des PECO (1997-2000) -}

\begin{tabular}{|c|c|c|c|}
\hline & $\begin{array}{c}\text { Exposition extra- } \\
\text { UEM (1) }\end{array}$ & & $\begin{array}{c}\text { Exposition } \\
\text { multilatérale (2) }\end{array}$ \\
\hline Bulgarie & 2,7 & Pologne & 7,8 \\
\hline Pologne & 3,3 & Roumanie & 10,0 \\
\hline Roumanie & 4,3 & Hongrie & 18,0 \\
\hline Hongrie & 6,4 & Lituanie & 19,8 \\
\hline Tchéquie & 9,3 & Bulgarie & 22,3 \\
\hline Slovénie & 9,3 & Lettonie & 22,9 \\
\hline Lituanie & 13,2 & Tchéquie & 23,0 \\
\hline Slovaquie & 16,1 & Slovénie & 24,2 \\
\hline Lettonie & 16,3 & Slovaquie & 34,7 \\
\hline Estonie & 24,8 & Estonie & 39,9 \\
\hline
\end{tabular}

(1) Produit de l'indicateur de symétrie des destinations exportatrices (cf. encadré 2) par le taux d'exportation extra-UEM d'un PECO exprimé en pourcentage. Moyenne annuelle sur la période.

(1) Produit de l'indicateur de symétrie des destinations exportatrices (cf. encadré 2) par le taux d'exportation multilatéral d'un PECO exprimé en pourcentage. Moyenne annuelle sur la période.

\footnotetext{
${ }^{9}$ Calculs de l'auteur à partir de CHELEM.
} 


\section{III- Les pays les plus exposés (Bulgarie, Estonie, Lettonie, Slovaquie) ont-ils intérêt à reporter leur adhésion à l’UEM ?}

\section{Une convergence réelle avec la zone euro?}

Les résultats des deux indicateurs d'exposition aux chocs (partie 1) et les mesures d'impact potentiel des chocs sectoriels et géographiques (partie 2) révèlent des asymétries importantes, notamment pour la Bulgarie et la Slovaquie, et plus encore pour l'Estonie et la Lettonie. Ces pays pourraient de ce fait avoir intérêt à reporter l'adhésion à l'union monétaire ou à se doter préalablement d'un fonds de stabilisation conjoncturel. Mais pour porter un tel jugement, il convient de prendre en compte tous les aspects de leur convergence réelle avec l'UEM, et non pas seulement celui de la symétrie des chocs de demande externe par secteur et destination. Les études portant sur la symétrie des chocs d'offre et de demande et sur la synchronisation des cycles entre les PECO et la zone euro constituent un bon support d'évaluation de la convergence réelle, et par voie de conséquence du caractère plus ou moins optimal de la zone monétaire en constitution. Les séries statistiques, encore insuffisamment longues, conduisent toutefois à des résultats peu robustes (J. Babetsky, 2003).

Rares sont les études couvrant l'ensemble des dix PECO adhérant à l'UE en 2004 ou 2007, et qui permettent donc de les comparer avec une méthode d'évaluation homogène. Nous ne nous référerons ici qu'aux études incluant simultanément les 4 pays mentionnés. D. Boreiko (2002) considère la corrélation des composantes cycliques de la production industrielle mensuelle entre les PECO et l'Allemagne. Elle enregistre une nette amélioration au cours de la période qui va de 1993 à 2001 pour l'Estonie et la Slovaquie, contrairement à la Lettonie et la Bulgarie - pour laquelle le lien devient négatif en fin de période. J. Fidmurc et I. Korhonen (2001) examinent, à partir d'un modèle structurel VAR, la corrélation des chocs d'offre et de demande avec l'ensemble de la zone euro, et concluent à une liaison positive sur la période 1994-2000 pour l'Estonie. Elle est alternativement positive et négative pour les trois autres économies selon le type de choc. A partir d'une méthode similaire et en raisonnant par rapport aux deux plus grandes économies de la zone euro, M. Frenkel, C. Nickel et G. Schmidt (1999) trouvent les corrélations des chocs d’offre les plus élevées entre l'Estonie et l’Allemagne et entre la Slovaquie et la France (période 1992-98).

Les cycles et les chocs letton et bulgare ne semblent donc pas converger avec ceux des économies de la zone euro. L'évolution de leur revenu par tête à long terme (ECB, 2004) montre que le processus de rattrapage avec celui de la zone euro n'en est qu'à ses prémisses. Il y a donc là matière à retarder l'entrée dans l'UEM jusqu'à ce qu'un processus de convergence réelle se soit véritablement enclenché. Cette recommandation s'adresse plus spécifiquement à la Lettonie, dont les autorités se sont prononcées en faveur d'une entrée dans le MCE 2 dès le $1^{\mathrm{er}}$ janvier 2005, en vue d'une adhésion à la zone euro à partir du $1^{\mathrm{er}}$ janvier 2007. On pourrait rétorquer que la Bulgarie et la Lettonie, qui ont adopté respectivement un ancrage-euro dans le cadre d'un currency board et un ancrage-DTS, n'ont nul besoin de conserver l'outil du taux de change. Le système de change bulgare est cependant trop récent (printemps 1997) pour pouvoir juger de sa soutenabilité. L’ancrage letton repose sur un taux fixe inchangé depuis février 1994 et sur une marge de fluctuation respectée de plus ou moins $1 \%$. Mais l'appréciation réelle du lats, due à un différentiel d'inflation accumulé avec les pays industrialisés depuis une dizaine d'années, laisse planer un doute sur la solidité de l'ancrage nominal (M. Bitans, 2002). De surcroît, l'euro ne comptant que pour 29,1 \% dans la composition du DTS, le taux de change du lats en euro reste volatile sur la période 1994-2001 (J. Babetsky, 2003). 


\section{Une réduction endogène de l’asymétrie des chocs de demande externe?}

L'idée d'un report de l'entrée dans l'UEM faute de convergence réelle ex-ante est contraire à l'approche endogène de la théorie des ZMO. J. A. Frankel et K. Rose ont été les premiers à avancer l'idée d'une convergence endogène ex-post. Ils montrent au travers d'études empiriques que l'usage d'une même monnaie est à l'origine d'un renforcement significatif des liens commerciaux (A. K. Rose, 2000), lui-même étant à l'origine d'une meilleure synchronisation des cycles (J. A. Frankel et K. Rose, 1998). La formation d'une union monétaire ou l'adhésion à une union créerait ainsi les conditions optimales de sa réussite, en réduisant de façon endogène l'asymétrie des chocs. Une hypothèse implicite mais non testée par les deux auteurs est que l'intensification des liens commerciaux reflète principalement une augmentation des échanges intrabranches. La réduction de l'asymétrie des chocs qui en découle induit une meilleure synchronisation des cycles. P. Krugman (1993) soutient la thèse inverse: les unions monétaires, à l'image de celle des Etats-Unis, développent les spécialisations interbranches, ce qui peut rendre une union non optimale ex-post, même si elle l'est ex-ante.

Plusieurs études viennent corroborer la thèse de Frankel et Rose dans le cas des économies européennes. L. Fontagné (1999), à l'aide d'une estimation en panel, montre que le passage à la monnaie unique devrait renforcer l'échange intrabranche au sein de l’Union européenne. Plus précisément, la baisse de l'interbranche devrait être compensée à parts égales par un accroissement des commerces intrabranches horizontale et verticale, d'où au total une baisse de l'intensité de la spécialisation intra-européenne. J. Fidrmuc (2001) met en évidence une relation significative entre le degré de commerce intrabranche et la corrélation des cycles des économies européennes avec le cycle allemand (période 1990-99), y compris pour les économies des cinq PECO (Hongrie, Pologne, République tchèque, Slovaquie et Slovénie) inclus dans son étude. L'étude de J. Babetski (2003), effectuée à partir d'un modèle VAR et portant sur l'ensemble des PECO (Bulgarie et Lituanie exceptées), révèle un lien entre l'intensité du commerce et la symétrie des chocs de demande de chacun des pays, aussi bien avec l’Allemagne que l’UE (période 1994-2002).

P. Kenen (2000) relance le débat sur la relation de cause à effet entre liens commerciaux et corrélation des cycles établie par J. Frankel et K. Rose, et qui s'expliquerait en bonne partie par une réduction de l'asymétrie des chocs. A partir d'un modèle keynésien à trois économies, P. Kenen montre que des liens commerciaux plus denses réduisent l'asymétrie d'un choc dérivé d'une chute de la demande d'un partenaire d'une même zone, compte tenu de la transmission s'opérant par le canal commercial. Mais il met en évidence d'autres types de chocs (comme un déplacement de la demande d'un pays tiers d'un partenaire vers un autre) pour lesquels l'intensification des liens commerciaux ne réduit pas l'asymétrie. Le caractère endogène de l'optimalité des unions monétaires devrait donc être relativisé, puisque la convergence des cycles dépend aussi de chocs dont le degré de symétrie est indépendant des liens commerciaux bilatéraux. Sans être étudié dans P. Kenen (2000), les deux types de chocs que nous mettons en avant dans ce travail ne dépendent pas principalement des liens commerciaux bilatéraux, à moins de considérer un choc dérivé d'une chute de la demande d'un pays membre de la zone euro. L'asymétrie d'un choc de demande externe sectoriel ou géographique dépend de la concentration différenciée des structures d'exportation d'un PECO et de l'UEM et de l'ouverture commerciale du PECO. 
Dans l'approche endogène de l'optimalité des unions monétaires, l'augmentation de la part de commerce intrabranche est considérée comme un réducteur d'asymétrie des chocs et de divergence des cycles. Les données dont nous disposons montrent que la hausse de la part du commerce intrabranche entre un PECO et l'UEM ne correspond pas nécessairement à une moindre exposition aux chocs asymétriques externes. Prenons le cas des chocs sectoriels potentiels. Nous avons fait observer en première partie que la hausse d'un indicateur de Grubel-Lloyd ne s'accompagnait pas forcément d'une convergence des structures d'exportation multilatérales. Par répercussion, et avec des taux d'exportation augmentant d'une période à l'autre, cette hausse n'implique pas de diminution obligée de l'exposition aux chocs asymétriques sectoriels (cf. tableau 5). L’idée d'une réduction endogène de l'asymétrie des chocs ne peut donc pas s’appliquer dans ce cas de figure. Parmi les 4 pays mentionnés cidessus, seule la Lettonie connaît une hausse de la part de son commerce intrabranche et intrazone de la période 1993-1996 à la période 1997-2000. Elle n'enregistre pas pour autant une meilleure symétrie de la structure de ses exportations par branche avec l'UEM. Le risque de choc asymétrique externe se trouve même accentué.

\section{Tableau 5 Evolution comparée du commerce intrabranche PECO-UEM et de l'indicateur d'exposition aux chocs sectoriels asymétriques}

- Moyenne annuelle par sous-période -

\begin{tabular}{|l|c|c|c|c|}
\hline \multirow{2}{*}{} & \multicolumn{2}{|c|}{$\begin{array}{c}\text { Indicateur de Grubel-Lloyd corrigé du } \\
\text { solde commercial (1) }\end{array}$} & \multicolumn{2}{c|}{$\begin{array}{c}\text { Indicateur d'exposition aux chocs } \\
\text { sectoriels asymétriques (2) }\end{array}$} \\
\cline { 2 - 5 } & $\mathbf{1 9 9 3 - 1 9 9 6}$ & $\mathbf{1 9 9 7 - 2 0 0 0}$ & $\mathbf{1 9 9 3 - 1 9 9 6}$ & $\mathbf{1 9 9 7 - 2 0 0 0}$ \\
\hline Lettonie & 0,27 & 0,38 & 24,7 & 28,5 \\
\hline Lituanie & 0,31 & 0,45 & 18,5 & 16,6 \\
\hline Roumanie & 0,45 & 0,47 & 10,5 & 13,4 \\
\hline Bulgarie & 0,55 & 0,48 & 17,8 & 19,6 \\
\hline Estonie & 0,60 & 0,58 & 17,3 & 36,5 \\
\hline Slovaquie & 0,61 & 0,61 & 14,3 & 16,7 \\
\hline Hongrie & 0,72 & 0,67 & 8,6 & 17,0 \\
\hline Pologne & 0,56 & 0,69 & 6,6 & 6,2 \\
\hline Slovénie & 0,73 & 0,75 & 14,1 & 14,3 \\
\hline Tchéquie & 0,77 & 0,81 & 9,7 & 13,1 \\
\hline SOne
\end{tabular}

Source : calculs de l'auteur effectués à partir des 71 branches de la base de données CHELEM

(1) Part du commerce intrabranche dans le commerce bilatéral total entre un PECO et l’UEM. La méthode de calcul de l'indicateur corrigé du solde figure en annexe.

(2) Sont reproduits ici les résultats du tableau 1. Une augmentation de l'indicateur traduit une plus forte exposition aux chocs. 


\section{Conclusion}

Les résultats obtenus témoignent d'une exposition généralement prononcée des 10 PECO aux chocs asymétriques externes de demande vis-à-vis de l'UEM. Six d'entre eux enregistrent une exposition plus marquée aux chocs sectoriels ou géographiques que celle de la Finlande, dont les structures d'exportation sont pourtant les plus concentrées de la zone euro après celles de l'Irlande. La Pologne figure pour les deux types de choc parmi les tout premiers pays classés, ce qui s'explique beaucoup plus par la faiblesse de son taux d'ouverture que par une convergence de ses structures d'exportation avec la zone euro. La prise en compte du taux d'exportation multilatéral et non plus seulement extra-UEM pour le calcul de l'exposition aux chocs géographiques ne modifie sensiblement le classement que pour la Bulgarie, compte tenu d'une forte concentration de ses ventes vers la Grèce et l'Italie. Les résultats d'indicateurs d'exposition aux chocs et les mesures d'impact potentiel par branche / destination révèlent des asymétries importantes pour la Bulgarie et la Slovaquie, et plus encore pour l'Estonie et la Lettonie.

Le processus de convergence réelle de la Bulgarie et de la Lettonie avec l'eurozone ne semblant pas être amorcé, ces deux économies pourraient envisager de reporter leur participation à l'UEM ou de créer préalablement un fonds de stabilisation conjoncturel, comme l'a fait la Finlande en $1997^{10}$. Cette recommandation entre en contradiction avec l'approche endogène de l'optimalité des unions monétaires. Cette approche considère que l'intensification du commerce intrabranche est un réducteur d'asymétrie des chocs et de divergence des cycles. Ce raisonnement ne doit pas être appliqué aux chocs sectoriel et géographique de demande externes : les données dont nous disposons montrent que la hausse de la part des échanges intrabranches entre un PECO et l'UEM ne correspond pas nécessairement à une moindre exposition à ces chocs. Ainsi la Lettonie, dont le commerce intrabranche s'intensifie au cours de la période 1993-2000, enregistre simultanément une plus grande vulnérabilité aux chocs asymétriques sectoriels.

\footnotetext{
${ }^{10}$ Nous mentionnons cette solution purement nationale, compte tenu de l'absence de consensus entre Etats membres sur la création d'un mécanisme budgétaire d'ajustement automatique à l'échelle de l'UEM (A. Italianer A. and M. Vanheukelen, 1993).
} 


\section{Bibliographie}

Babetsky J. (2003), "EU Enlargement and Endogeneity of OCA Criteria: Evidence from the CEECs”, ROSES, Conference on Enlargement Economics.

Babetsky J., Boone L., Maurel M. (2002), "Exchange Rate Regimes and Supply Shocks Asymmetry: the Case of the Accession Countries”, CEPR Discussion Paper, DP3408, June.

Bayoumi T., and Eichengreen B. (1993), "Shocking Aspects of European Monetary Unification", in The Transition to Economic and Monetary Union in Europe, ed. by F.Giavazzi and F. Torres, Cambridge University Press.

Bayoumi T., and Eichengreen B. (1994), “One Money or Many?”, Princeton Studies in International Finance, $\mathrm{n}^{\circ} 76$, Princeton University Press.

Bayoumi T., and Eichengreen B. (1997), "Ever closer to heaven? An optimum-currency areaindex for European countries”, European Economic Review, 41.

Bénassy-Quéré A. et Lahrèche-Révil A. (1998), "Pegging the CEECs currencies to the euro ", Document de travail, CEPII, $\mathrm{n}^{\circ} 4$.

Bitans M. (2002), « Real Exchange Rate in Latvia (1994-2001) », Bank of Latvia.

Boone L. et Maurel M. (1999), «L'Ancrage de l'Europe centrale et orientale à l'Union européenne », Revue économique, vol. 50, n 6, novembre.

Boreiko D. (2002), "EMU and Accession Countries: Fuzzy Cluster Analysis of Membership”, ONB, WP 71.

ECB - European Central Bank (2004), “The Acceding Countries' Economies on the Threshold of the European Union”, Monthly Bulletin, February.

Eichengreen B. (1994), “The Optimum Currency Dilemma”, International monetary arrangements for the $21^{\text {st }}$ century - Integrating national economies, The Brookings Institution.

Fidrmuc J. (2001), “The Endogeneity of Optimum Currency Area Criteria, Intra-industry Trade and EMU Enlargement”, BOFIT Discussion paper, $n^{\circ}$ 8, Bank of Finland, Institute for Economies in Transition.

Fidmurc J., and Korhonen I. (2001), "Similarity of Supply and Demand Shocks between the Euro Area and the CEECs", BOFIT Discussion Paper, n ${ }^{\circ} 14$, Bank of Finland, Institute for Economies in Transition.

Finger J. M., and Kreinin M. E. (1979), “A Measure of Export Similarity and its Possible Uses“, The Economic Journal, vol. 89, n³ 356, December.

Fontagné L. (1999), "Spécialisation et asymétrie des chocs en union monétaire ", Revue française d'économie, volume XIV, 2.

Fontagné L. et M. Freudenberg M. (1999), “ Marché unique et développement des échanges ”, Economie et statistique, $\mathrm{n}^{\circ} 326-327$.

Frankel J. A. (1999), « No Single Currency Regime is Right for All Countries or All Times », NBER Working Paper, $\mathrm{n}^{\circ}$ 7338, september.

Frankel J. A., and Rose A. K. (1998), "The Endogeneity of the Optimum Currency Area Criteria ”, The Economic Journal, vol. 108, n 449, july.

Frenkel M., Nickel C., and Schmidt G. (1999), "Some Shocking Aspects of EMU Enlargement”, Deutsche Bank Research Note, $\mathrm{n}^{\circ} 4$.

Freudenberg M. et Lemoine F. (1999), «Les Pays d'Europe centrale et orientale dans la division du travail en Europe ", Economie internationale, $n^{\circ} 80,4^{\text {ème }}$ trimestre. $^{2}$

Grubel H., and Lloyd P. (1975), Intra-Industry Trade, MacMillan. 
Ishiyama Y. (1975), “The theory of optimum currency areas: a survey”, International monetary fund staff papers, 22.

Italianer A., and Vanheukelen M. (1993), «Proposals for community stabilization mechanisms: some historical applications ", European economy, "The Economics of community public finance ", Reports and studies, $\mathrm{n}^{\circ} 5$.

Kenen P. (1969), “The Theory of optimum currency areas : an eclectic view”, Monetary problems of the international economy, R. Mundell and K. Swoboda, The University of Chicago press.

Kenen P. (2000), "Currency Areas, Policy Domains, and the Institutionalization of Fixed Exchange Rates”, mimeo, Princeton University.

Krugman P. (1993), "Lessons of Massachusetts for EMU”, in F. Torres and F. Giavazzi Eds, Adjustment and Growth in the European Monetary Union, Cambridge, Cambridge University Press.

Kucerova Z. (2003), “The OCA Theory and its Application to Central and Eastern European Countries”, Department of Macroeconomics, Technical University of Ostrava.

McCallum B. T. (1999), « Theorical Issues Pertaining to Monetary Unions », NBER Working Paper Series, 7393.

MacKinnon R. I. (1963), “Optimum currency areas”, American economic review, vol. 53, june.

Masson P. R., and Taylor M. P. (1993), "Currency Unions: a Survey of the Issues », in Policy issues in the operation of currency unions, Cambridge University Press.

Mongelli F. P. (2002), "New views on the optimum currency area theory: what is EMU telling us?”, Working paper, European central bank, n 138, April.

Mundell R. A. (1961), “A Theory of Optimum Currency Areas ”, American economic review, vol. 51.

Rose A. K. (2000), “One money, one market: estimating the effect of common currencies on trade ”, Economic policy, February, Forthcoming.

Tavlas G. (1993), “The "New” Theory of Optimum Currency Areas”, World Economy, 16 (6), November. 


\section{ANNEXE Calcul de l'indicateur de Grubel-Lloyd corrigé du solde commercial}

Le calcul des échanges intrabranches s'effectue traditionnellement à partir de l'indicateur de Grubel et Llyod suivant :

$$
G L=1-\frac{\sum\left|X_{i}-M_{i}\right|}{\sum\left(X_{i}+M_{i}\right)} \text {, pour i variant de } 1 \text { à n }
$$

Où $\mathrm{X}_{\mathrm{i}}$ et $\mathrm{M}_{\mathrm{i}}$ sont respectivement les exportations et les importations de la branche $\mathrm{i}$.

En cas de déficit ou d'excédent commercial, une partie des importations ou des exportations n'est pas compensée par un flux réciproque. Or, au sens strict, pour parler d'échange intrabranche (ou interbranche), il faut avoir un croisement de produits similaires (ou différents). Le solde commercial fausse donc le calcul des parts des commerces intrabranche et interbranche. La part du commerce intrabranche calculée par GL sera d'autant moins élevée que le déséquilibre commercial est important. L’indicateur est donc biaisé vers le bas. On peut corriger ce biais en calculant l'indicateur GL', d'où sont issus les résultats du tableau 5 :

$$
\mathrm{GL}^{\prime}=\mathrm{GL} /(1-\mathrm{k})
$$

$$
\text { Avec : } \quad k=\frac{\left|\sum X_{i}-\sum M_{i}\right|}{\sum\left(X_{i}+M_{i}\right)}
$$

Si le solde extérieur (numérateur) est nul, $\mathrm{k}=0$ et GL = GL'. Plus le solde extérieur est élevé, plus k est proche de 1 et GL’ différent de GL. 